\title{
Ultrashort pulse control of space-dependent excitations in a three-level system
}

\author{
Yu. Loiko, ${ }^{1,4}$ C. Serrat, ${ }^{1}$ R. Vilaseca, ${ }^{1}$ V. Ahufinger, ${ }^{2,3}$ J. Mompart, ${ }^{2}$ and R. Corbalán ${ }^{2}$ \\ ${ }^{1}$ Departament de Física i Enginyeria Nuclear, Universitat Politècnica de Catalunya, Colom 11, 08222 Terrassa, Spain \\ ${ }^{2}$ Departament de Física, Universitat Autònoma de Barcelona, 08193 Bellaterra, Spain \\ ${ }^{3}$ ICREA-Institució Catalana de Recerca i Estudis Avançats, Barcelona, Spain \\ ${ }^{4}$ Institute of Molecular and Atomic Physics, National Academy of Sciences of Belarus, Nezaleznasty Ave. 70, 220072 Minsk, Belarus
}

(Received 10 October 2006; revised manuscript received 12 December 2006; published 1 February 2007)

\begin{abstract}
Coherent control of regions with spatial excitation of populations and coherence between two lower states in three-level $\Lambda$-type quantum systems mediated by the self-induced transparency (SIT) phenomenon is theoretically investigated with one- and two-color ultrashort pulses and with pulse sequences beyond the slowly varying envelope (SVEA) and rotating wave (RWA) approximations. The effects of different parameters characterizing the pulses and the medium on the location as well as the width of such excited regions have been studied numerically by means of the finite-difference time-domain (FDTD) method. It has been determined that with a scheme of two-pulse excitation one can effectively control the position at which the region is written and its width. In particular, the position of the excited region can be controlled by the area of the pulses. We find that the maximum value of the population transferred to the lower excited state depends on the detuning of the pulses with respect to the one-photon resonances, and that both position and width of the region also depend on the temporal duration of the pulses. We show how after the excited region is written, its position can be shifted by additional pulses. On the basis of numerical results, scaling laws are formulated for the reported phenomena. With such control, the width of the region excited inside the medium can be reduced to the order of the wavelength of the light.
\end{abstract}

DOI: 10.1103/PhysRevA.75.023801

PACS number(s): 42.50.Gy, 42.50.Md, 42.65.Re

\section{INTRODUCTION}

It is known that in three-level quantum systems two types of form stable pulse propagation are possible, which can be distinguished on the basis of dynamics in the dressed state representation [1,2]. Such unperturbed propagation of pulses can be caused by phenomena either of self-induced transparency (SIT) $[3,4]$ or electromagnetically induced transparency (EIT) [5-7].

Recently, trapping, storage, and release of light pulses in and out from collective medium excitations in a three-level $\Lambda$-type system by the dynamical EIT effect have been proposed theoretically [8] and demonstrated experimentally in gaseous $[9,10]$ and in solid state media [11,12] (Pr:YSO). One of the key points of light storage with the EIT effect is the reduction of the group velocity of the pulse to be stored, in the presence of an additional external control field. It is well known that the decrease of light group velocity can be realized beyond the EIT condition too, for instance by SIT phenomena. Previously, the possibility of providing distinct domains with spatial excitation of the two lower states by SIT pulses in a three-level $\Lambda$-type system was reported in Ref. [13] under the slowly varying envelope (SVEA) and rotating wave (RWA) approximations, and in Ref. [14] beyond these approximations. In the present study we address the problem of coherent control (creation, manipulation and reading) of such excited regions by means of the SIT effect.

The utilization of the EIT effect for effective storage of light pulses has some restrictions [8,15-17]. One of them is that the bandwidth (inverse temporal duration) of the pulse to be stored cannot exceed the spectral width of the transparency window induced by the EIT control field. Another one is that the temporal duration of the pulse to be stored should be larger than the decay times of the quantum states in the medium, in order to perform the coherent population trapping. Therefore, the speed of storage, manipulation and reading of light pulses provided by such scheme is limited by the temporal duration of the pulse to be stored, by the strength of the control field (by which the bandwidth of the transparency window is established) and by the minimum relaxation times of the quantum states in the medium. In experiments with EIT storage of light pulses in solid-state media, see for instance Refs. [11,12], large intensities are needed, which means that the area of the pulses becomes of the order of $\pi$ and that the effects of SIT phenomena become of great importance. Moreover, as it was shown in Ref. [17], when the amplitude of the probe pulse (which is stored in the medium) becomes comparable with that of the control field, the spectral width of the EIT window becomes narrower, which results in significant absorption of the probe pulse and in temporal broadening. Therefore, the retrieval pulse becomes distorted. Results from recent experiments [18] indicate that a GHz bandwidth of storage speed can be feasible with EITbased techniques.

In this paper we show that with the SIT phenomenon under ultrashort pulse excitation there are no lower limits on the time duration of the stored pulses and, therefore, one can provide optical storage at higher speed than that provided with the EIT storage technique of Ref. [8]. Moreover, with SIT pulse storage one can enhance the light pulse writing process by applying an additional $\mathrm{cw}$ or pulsed coupling field at the frequency of the transition uncoupled from the ground state [the transition $|1\rangle \rightarrow|2\rangle$ in Fig. 1(a), of frequency $\omega_{12}$ ], which reinforce the stimulated coherent processes in the system.

Here we investigate in detail the possibilities for coherent control of the spatial position (location) and width of coher- 


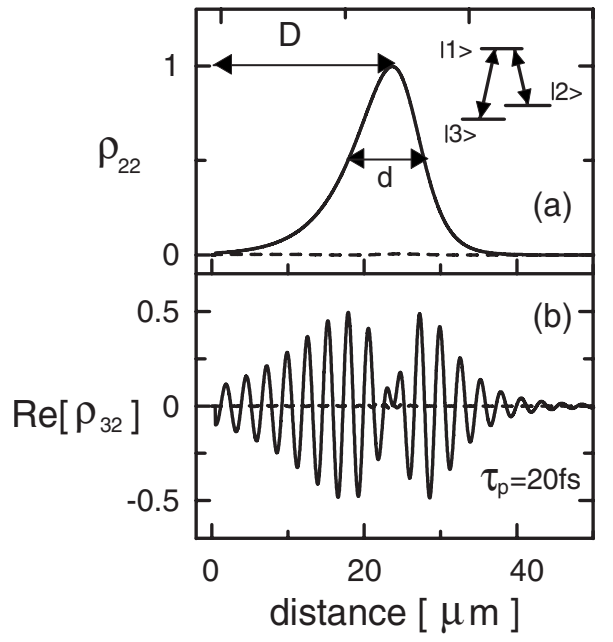

FIG. 1. (a) Spatial distribution of the population of the lower excited level $\rho_{22}$ of a three-level $\Lambda$-type system with the level numbering indicated in the inset. (b) Instantaneous spatial distribution of the oscillations in the real part of the coherence $\rho_{32}$ between levels $|2\rangle$ and $|3\rangle$ (solid line) and of the coherences $\rho_{31}$ and $\rho_{21}$ (dashed lines). The amplitudes of the oscillations of the coherences $\rho_{31}$ and $\rho_{21}$ are very small and almost undistinguishable from the zero level in (b). The excitations of population and coherences are induced in the medium (see the text for parameters) by two-color $(2 \pi, 0.3 \pi)$ pulses with time duration $\tau_{p}=20$ fs. The first of these pulses has frequency $\omega_{1}$ which is in resonance with the $|1\rangle \rightarrow|3\rangle$ transition and its area is equal to $2 \pi$. The area of the second pulse is equal to $0.3 \pi$ and its frequency $\omega_{2}$ is in resonance with the $|1\rangle \rightarrow|2\rangle$ transition. The centers of the pulses are initially aligned in time.

ently excited regions in a three-level $\Lambda$-type medium by ultrashort one- and two-color laser pulses of SIT type, whose time duration is less than the characteristic relaxation times in the system, i.e., only coherent processes are involved. In particular, we provide a numerical study on the effects of atom density, pulse detuning from one-photon resonance conditions, pulse area and time duration of pulses in one spatial dimension. We utilize the full set of Maxwell and density matrix equations beyond the RWA and the SVEA approximations [14] in order to include all the nonlinear processes that could influence the phenomena under consideration and to account in the same way the optical field tuning close and far from the resonances of the optical transitions, since the ultrashort pulses that we investigate have wide spectral bands which overlap with different transitions of the medium. Furthermore, we consider ultrashort intense pulses, and therefore as it has been shown previously [19], in order to properly account for the carrier frequency changes and reshaping of the pulses one needs to perform calculations beyond the RWA and the SVEA approximations. In this study the transitions of the medium are assumed to be homogeneously broadened, although the results that we obtain could also be tested in inhomogeneously broadened media since the SIT phenomenon is also present in such systems [3].

The other fundamental question of particular interest in theory and in possible applications of light pulse storage for optical information processing which we address in this pa- per, is on which spatial and time scales such writing, manipulation and reading can be achieved. The scaling laws formulated here on the basis of numerical simulations provide an answer to this question for three-level $\Lambda$-type systems with excitation by SIT pulses or pulse sequences. In particular, we study how one can predict excitation of a medium by a pulse if the excitation provided in the same medium by pulses with other temporal durations and/or amplitudes is known. In our numerical simulations, we identify the conditions under which pulses with different temporal durations provide similar spatial distributions of state excitations (level populations and coherences) inside the medium. Under these conditions, scaling laws are formulated for the locations and the spatial widths of the regions where light pulses are stored. Additionally, the obtained scaling laws are applied to show that excitation of level populations and coherences in a three-level $\Lambda$-type system can be achieved in a spatial domain, in particular, of the order and even less than the light wavelength. Moreover, the scaling formulated here can be considered as a complementary tool for theorems formulated previously such as the area theorem by McCall and Hahn [3].

The paper is organized as follows. In Sec. II the control of the spatial width and the position of a region with transfer of population and excitation of coherence between the two lower levels in a three-level $\Lambda$-type quantum system by oneand two-color pulses is considered in one spatial dimension. Scaling laws and scaling conditions under which these laws are realized, for interactions of a three-level $\Lambda$-type system with single (one-or two-color) pulses and with pulse sequences are presented in Secs. III and IV, respectively. In Sec. IV we also discuss possibilities for coherent control (movement and reading) of a previously excited region by pulse sequences. In Sec. V we summarize the obtained results.

\section{DEPENDENCE OF THE EXCITED REGION ON DIFFERENT PARAMETERS}

In a previous paper [14], some of us discussed the formation of excited regions inside a three-level $\Lambda$-type medium, where population from the ground level $|3\rangle$ [see, the inset in Fig. 1(a)] was transferred to the lower excited state $|2\rangle$ while pulses propagated through the system. In this section we investigate the control of this excited region by studying the effects of different parameters on its position and width.

We consider propagation of laser pulses through a homogeneously broadened medium filled with three-level quantum systems (atoms or molecules) of $\Lambda$-type [see the inset in Fig. 1(a)] as described by the model presented in the Appendix. This $\Lambda$-system can interact with electromagnetic radiation through two allowed (under the electric-dipole approximation) transitions with frequencies $\omega_{12}$ and $\omega_{13}$ [the numbering of quantum states is depicted in the inset in Fig. 1(a)]. We will consider propagation of one-or two-color hyperbolic secant (HS) pulses, i.e., we will assume that the input pulse has one or two spectral components. The central frequencies of the input pulses are taken in resonance with the transitions $|1\rangle \rightarrow|3\rangle$ and $|1\rangle \rightarrow|2\rangle$, and are denoted by $\omega_{1}$ and $\omega_{2}$, respectively. Initial pulse areas are denoted by $A_{1}$ 
and $A_{2}$. In what follows, the notation $(m \pi, n \pi)$ is used for pulses with $m \pi$ area of the pulse at $\omega_{1}$ and $n \pi$ area of the pulse at frequency $\omega_{2}$. The pulses are considered initially aligned in time. We will use the notation $\tau_{p}$ for the full width at half-maximum (FWHM) of the pulse intensity envelope. For definiteness and simplicity, the time durations of the pulses $\omega_{1}$ and $\omega_{2}$ are considered equal with each other, and the ratio of frequencies of optical transitions is fixed at $\omega_{12} / \omega_{13}=0.7$. The angular frequency of the transition $|1\rangle$ $\rightarrow|3\rangle$ is taken as $\omega_{13}=2.36 \times 10^{15} \mathrm{~s}^{-1}$ (which corresponds to a wavelength such as $\lambda_{13}=0.8 \mu \mathrm{m}$ ). Dipole moments of both transitions are equal with each other. The medium is assumed to be initially prepared in the ground state [state $|3\rangle$ in Fig. 1(a)]. The value $N=2 \times 10^{24} \mathrm{~m}^{-3}$ for concentration of active centers (atoms or molecules) in the medium will be used. Solid-state crystals [11] can be considered as active media under the investigations presented here. We disregard the relaxation processes in the medium, because time durations of the pulses are less than any relaxation times of the system. We will assume that each pulse can interact with both transitions, therefore, each pulse can induce variations in any transition of the system. Since the $\omega_{1}\left(\omega_{2}\right)$ pulse is far from resonance with the $\omega_{12}\left(\omega_{13}\right)$ transition, the variation induced by this pulse in this transition will be much smaller than the one induced by the $\omega_{2}\left(\omega_{1}\right)$ pulse. The details of the model and the finite-difference time-domain (FDTD) scheme for the numerical integration is described in the Appendix.

It has been previously reported $[13,14]$ that inside the considered three-level $\Lambda$-type system any $(m \pi, n \pi)$ pulse, in particular a $(2 \pi, 0)$ pulse, undergoes frequency downconversion with almost total conversion from the $\omega_{1}$ pulse into a pulse with frequency $\omega_{2}=\omega_{1}-\omega_{23}$. Simultaneously, population from the ground state $|3\rangle$ is effectively transferred to the lower excited state $|2\rangle$ in a short region, see Fig. 1(a). In what follows, we will name such region as excited region. This region is surrounded on both sides by regions with excited coherence between the two lower states [see Fig. 1(b)]. Such excited regions can be considered as regions in which information is stored. Under pulses with area larger than $2 \pi$, more than one of such regions can be excited in the medium, see Ref. [14], Fig. 2, and the discussion below.

Now we address the question of how one can control the position at which the excited regions appear and how one can modify their widths. For this purpose we investigate the excitation of a three-level $\Lambda$-type quantum system (initially prepared in the ground state $|3\rangle)$ with two-color pulses. One should note that the excitation could be provided solely by one-color $\omega_{1}$ pulse, as shown in Ref. [14]. In our simulation we use the second pulse with frequency $\omega_{2}$ only in order to reduce the distance at which the excited region appears. Therefore, hereafter we consider excitation of the medium with $(n \pi, m \pi)$ two-color pulses for $n \neq 0$ and $m \neq 0$. The other effect of the $\omega_{2}$ pulse is to provide initial coherence of the medium at the time when the $\omega_{1}$ pulse enters the medium. The same effect of the control field in the EIT scheme has been outlined in Ref. [15].

First we fix the temporal pulse duration at $\tau_{p}=40 \mathrm{fs}$ and investigate the effect of the pulse area $A_{1}$ of the $\omega_{1}$ pulse on the excitation region when the area of the $\omega_{2}$ pulse is fixed at

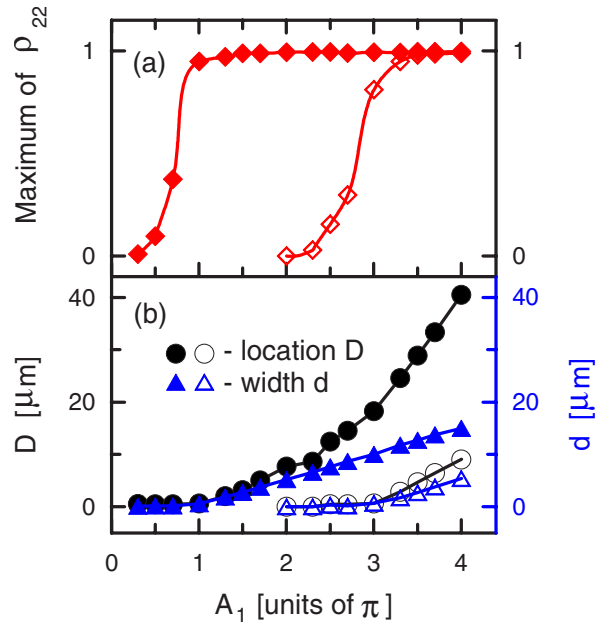

FIG. 2. (Color online) Dependence on the pulse area $A_{1}$ of the pulse with frequency $\omega_{1}=\omega_{31}$ (a) of the maximum value of the population of level $|2\rangle \rho_{22}$ (rhombuses); (b) the distance $D$ at which the maximum of $\rho_{22}$ is achieved in the region with population transfer and excited coherence (circles), and spatial width $d$ of this region (triangles). Excitation has been performed by two-color HS $\left(A_{1}, 0.3 \pi\right)$ pulses, whose frequencies are at resonance with the two transitions of the $\Lambda$-system. Temporal duration of these pulses is $\tau_{p}=40$ fs. Open rhombuses, circles, and triangles denote the corresponding characteristics of the second excited region that appears for $A_{1}>2 \pi$ as a result of the splitting of the initial $\omega_{1}$ pulse in a sequence of $2 \pi$ pulses and the remaining radiation with area less than $2 \pi$.

the value $A_{2}=0.3 \pi$. The results are presented in Fig. 2, where the maximum value of the population $\rho_{22}$ transferred to level $|2\rangle$ [Fig. 2(a)], and the distance at which the excited region is created and its width [Fig. 2(b)] is plotted as a function of the pulse area $A_{1}$. From these data it follows that $\omega_{1}$ pulses with area $\pi<A_{1}<2 \pi$ provide a single excited region (see closed rhombuses, circles, and triangles in Fig. 2). Pulses whose area is less than $\pi$ can provide only partial population transfer $\left(\rho_{22}<1\right)$ in the excited region (see Fig. 2). When the pulse area is larger than $2 \pi$, the excited region becomes divided in different subregions (see open and closed rhombuses, circles, and triangles in Fig. 2 for $A_{1}>2 \pi$ ). The maximum population $\rho_{22}$ in most of these regions is almost equal to 1 but there are subregions in which the maximum of $\rho_{22}$ is less than 1 . This behavior is expected since each pulse with area larger than $2 \pi$ is divided during propagation through the medium into a sequence of $2 \pi$ pulses and a remaining radiation whose area is less than $2 \pi$ (see Refs. $[3,4,14]$ ); the pulses with area $2 \pi$ write excitation regions with total population transfer at the central part of the region, but the remaining radiation, whose area is less than $2 \pi$, induces only partial population transfer, as it follows from the data presented in the domain $A_{1}<\pi$ in Fig. 2. In the case presented in Fig. 2 when $2 \pi<A_{1}<4 \pi$, the initial $\omega_{1}$ pulse is separated into a pulse with $2 \pi$ area, which writes the excited region with total population transfer at the center, and a remaining emission, which induces the appearance of an additional excited region with partial population transfer at the center. From Fig. 2 it also follows that increasing the pulse area $A_{1}$, the distance at which the excited region is written and the 


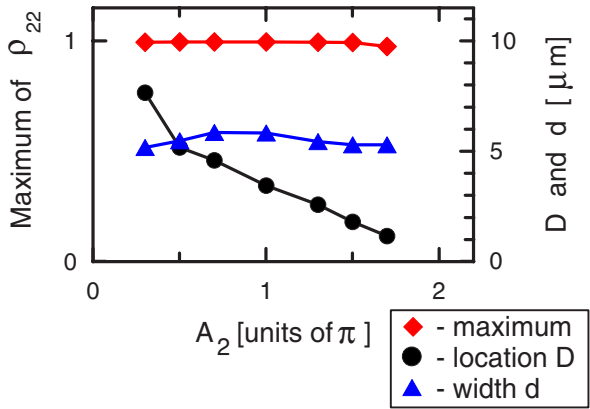

FIG. 3. (Color online) The same as in Fig. 2 but with dependence on the pulse area $A_{2}$ of the pulse with frequency $\omega_{2}=\omega_{12}$. Excitation has been performed by two-color $\left(2 \pi, A_{2}\right)$ pulses, whose frequencies are at resonance with the two transitions of the $\Lambda$-system. The notations are the same as in Fig. 2.

spatial width of this region are increased. The increase in the distance at which the excited region appears is attributed to the increase in the velocity of pulse propagation with increasing area (see Refs. $[3,4]$ ). Increase of the spatial width of the excited region, however, is caused by the increase of the number of photons in the pulse (increase in the pulse energy) with increasing the pulse area.

The frequency down-conversion of the pulse $\omega_{1}$ can be enhanced by changing the pulse area of the pulse at frequency $\omega_{2}$. An increase in the area of the pulse $\omega_{2}$ decreases the distance at which the excited region appears. For pulses with time duration $\tau_{p}=40 \mathrm{fs}$ this effect is shown in Fig. 3, where the dependence of the excited region characteristics on the area of the pulse $\omega_{2}$ is presented. This decrease in the distance at which the excited region appears is caused by stimulated emission in the transition $|1\rangle \rightarrow|2\rangle$ and by the stimulated Raman process in the transitions $|3\rangle \rightarrow|1\rangle \rightarrow|2\rangle$ in the presence of the pulse $\omega_{2}$. With increasing the area of the pulse $\omega_{2}$, these processes are enhanced. Because the spatial width of the excited region and the maximum value of the population in level $|2\rangle$ at its center are defined predominantly by the area of the pulse $\omega_{1}$, the changes in the area of the pulse $\omega_{2}$ do not influence significantly these characteristics, as it is clearly shown in Fig. 3.

From the preceding discussion it follows that the maximum value of the population of level $|2\rangle$ at the center of the excited region cannot be controlled effectively by the parameters considered above. We have found that such control as well as the control of the position and the spatial width of the excited region can be performed by detuning the pulse frequencies from the one-photon resonance, provided the twophoton resonance condition is kept. This possibility is illustrated in Fig. 4(a), where the dependence of the excited region characteristics on the detuning of two-color $(2 \pi, 0.3 \pi)$ pulses at fixed values of the other parameters is plotted. The maximum value of population of level $|2\rangle$ at the center of the excited region is decreased by increasing the detuning, which simultaneously increases the spatial width of the excited region and the distance at which such region is induced by the pulse $\omega_{1}$.

The concentration of active atoms (molecules) can also influence the considered phenomena. We have checked that the effect of population transfer and excitation of coherence
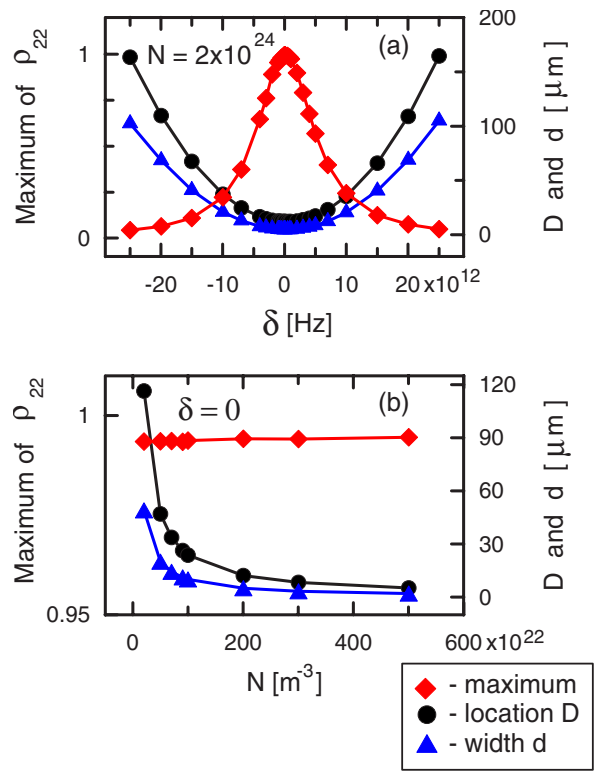

FIG. 4. (Color online) Effects of the detuning $\delta=\omega_{1}-\omega_{31}=\omega_{2}$ $-\omega_{21}$ of the pulse frequencies from the one-photon resonance (keeping the two-photon resonance condition) (a) and of the concentration $N$ of active centers in the medium (b) on the characteristics of the excited region, which are the maximum value of the population in level $|2\rangle$ (rhombuses) and the location $D$ (circles) and the spatial width $d$ (triangles) of the region. The $\Lambda$-system is excited by two-color $(2 \pi, 0.3 \pi)$ pulses with temporal duration $\tau_{p}=40$ fs. In (a) the concentration of active centers is assumed to be $N=2$ $\times 10^{24} \mathrm{~m}^{-3}$, and in (b) the pulses are tuned to the resonance with the corresponding transitions of the $\Lambda$-medium.

persist at lower concentrations. However, at low concentration (which is usually the case of experiments with gaseous media), the distance at which the excitation takes place and the width of the excited region increase noticeably, as shown in Fig. 4(b).

We have therefore shown that several parameters of the pulses and of the medium can influence all the characteristics of the excited region, namely, (i) its location in space, (ii) its spatial width, and (iii) the maximum population of level $|2\rangle$ at the center of the region. By acting on such parameters an effective control of the excited region can be achieved.

\section{TEMPORAL PULSE DURATION AND SCALING LAWS}

In this section we consider the temporal pulse duration as the main control parameter, and we present results on scaling properties of the light pulse interaction with a three-level $\Lambda$-type quantum system. Indeed, we show that pulses with different temporal durations and amplitudes but with the same pulse areas can provide excited regions with similar spatial distributions. On the basis of the numerical simulations of the considered system we formulate scaling laws, which reveal the relation between the pulse duration and the location and spatial width of the excited region.

We will illustrate the above outlined question with an example of the excitation of the three-level $\Lambda$-system with twocolor $(2 \pi, 0.3 \pi)$ pulses (introduced in the preceding section) 


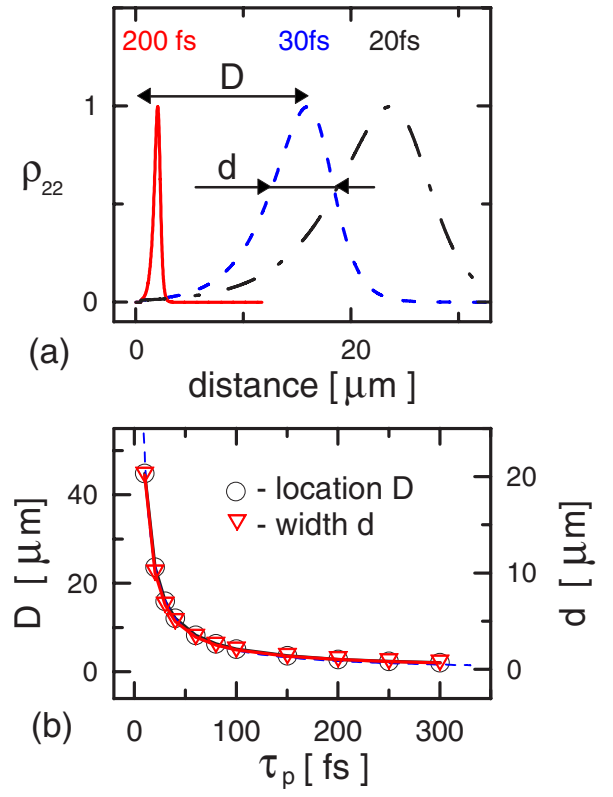

FIG. 5. (Color online) Scaling properties of the excitation of a three-level $\Lambda$-type quantum system. (a) Spatial distribution of population of level $|2\rangle$ for three different time durations of the injected $(2 \pi, 0.3 \pi)$ pulses. (b) Dependence of location distance $D$ (circles) and width $d$ (triangles) of the region with population transfer to the level $|2\rangle$, and excited coherence between the two lower levels, on the temporal width $\tau_{p}$ of the injected two-color $(2 \pi, 0.3 \pi)$ pulses. In (b), data obtained with the FDTD numerical integration of the model is represented by solid lines, whereas analytical curves obtained by the expressions (1) and (2) are depicted by the dashed lines. Note that in all cases the area of the pulses is kept constant, and that the components of the two-color pulses are in resonance with the corresponding transitions of the $\Lambda$-system.

at several values of temporal pulse durations by keeping constant the area of pulses. The medium is initially prepared in the ground state $|3\rangle$. The results are presented in Fig. 5. Fig. 5(a) shows the scaling behavior in the spatial distribution of population of level $|2\rangle$ in the excited region, which is induced by pulses with three different time durations. Figure 5(b) depicts how the distance of the excited region and its spatial width depend on the pulse duration. In Fig. 5(b), the numerical data obtained by the FDTD integration of the model are represented by solid lines and symbols. From these data we can derive the following scaling laws between the pulse duration $\tau_{p}$, the spatial location $D$ and the width $d$ of the excited region:

$$
\begin{aligned}
& D \times \tau_{p}=K_{1}, \\
& d \times \tau_{p}=K_{2} .
\end{aligned}
$$

where $K_{1}$ and $K_{2}$ are constants for a given medium.

Analytical curves described by Eqs. (1) and (2) are presented by dashed lines in Fig. 5(b). The analytical curves are indistinguishable from the numerical data, which clearly shows the validity of the $1 / \tau_{p}$ scaling laws for the spatial location $D$ and the width $d$ of the excited region. We have performed calculations for different values of the pulse area.
In a general sense, the presented scaling laws cannot be fulfilled if other effects come into play. Indeed, we have observed deviation from the results of the presented scaling laws for pulses whose area becomes of the order of $\pi$ or smaller. In that case, ordinary absorption dominates in propagation. The scaling laws are fulfilled however for pulses whose area is near and larger than $2 \pi$. This result is expected, since as it is well known, during the propagation in two- and three-level systems, a pulse whose area is larger than $2 \pi$, is reshaped into a $2 \pi$ pulse, or into a sequence of $2 \pi$ pulses. We have checked that this reshaping occurs much before the spatial excitation of the medium for singlefrequency pulses. In our simulations we find that each of these reshaped $2 \pi$ pulses undergoes frequency down conversion and writes an excited region in the medium, and that these written regions obey the scaling laws (1) and (2). Therefore, in the one-dimensional (1D) case we have demonstrated that under constant pulse area the distance and spatial width of the region excited by a pulse in a three-level $\Lambda$-system prepared in the ground state is inversely proportional to the temporal pulse duration.

Scaling laws (1) and (2) may be considered as complementary to the area theorem formulated by McCall and Hahn [3]. For instance, from this theorem it follows that the evolution and, therefore, the final state of a medium is defined by the area of the propagating pulses. From our scaling conditions derived for the $1 \mathrm{D}$ case it follows that pulses with the same pulse area but with different temporal durations and envelope amplitudes will induce different spatial distributions in the medium. These distributions are scaled in space with a factor that is inversely proportional to the ratio of temporal durations of the pulses.

From the analysis of Eqs. (1) and (2), it follows that a pulse with a larger temporal duration (i.e., smaller envelope amplitude) will excite a region inside the medium with a shorter spatial width and at shorter spatial distance than that with a smaller temporal duration (i.e., with a larger envelope amplitude). Therefore, by increasing the pulse duration (with simultaneous decrease in the envelope amplitude, under the condition of constant pulse area) the spatial width of the excited region can be made very small, for instance with a value smaller than the light wavelength. For the data presented in Fig. 5(b), the spatial width of the excitation region indeed becomes less than the light wavelength $(\lambda=800 \mathrm{~nm})$ for a temporal pulse duration above $200 \mathrm{fs}$. Such region is presented in Fig. 5(a). It should be noted that the values of the concentration of active atoms (molecules) which we used in the simulations presented here are higher than those that would normally be used in experiments with gaseous media, but they can still be met in solid-state and semiconductor media. Therefore, our simulations predict that in such threelevel media, it is possible to store a light pulse in the excitation of the populations of the medium and the coherences (i.e., to store information) in a region with a spatial scale smaller than the light wavelength.

\section{CONTROL OF THE EXCITED REGION WITH MULTIPLE PULSES}

In the preceding sections it has been shown how one can excite a region with given characteristics, in which a light 
pulse is mapped on the populations and the coherence of the two lower states, by exciting the medium with single (one- or two-color) laser pulses of the SIT type. In this section, we consider the problem of how, once the excited region has been created, the excited region characteristics, in particular its position, can be modified by means of subsequent light pulses. We also consider how one can extract the information stored in the system. Further, we will show that the scaling laws introduced above can also be extended to this more general case.

Let us consider writing and subsequent modification of a excited region by two sequences of laser pulses in the medium with length $L=17 \mu \mathrm{m}$. In each sequence three pulses are injected. The first pulse is a two-color $\left(\omega_{1}, \omega_{2}\right)$ pulse. It creates (writes) the excited region inside the medium. The second pulse is injected into the system after the writing $\left(\omega_{1}, \omega_{2}\right)$ pulse with a delay of $8 \tau_{p}$, and the last (third) pulse is injected with the same delay but with respect to the second pulse. The two last pulses are tuned at resonance with the transition $|1\rangle \rightarrow|2\rangle$ [see Fig. 1(a)], i.e., they have frequency $\omega_{2}=\omega_{12}$. All pulses in the sequence have the same time duration $\tau_{p}$. Let us call the pulses with frequency $\omega_{2}=\omega_{12}$ that come after the writing pulse as reading or controlling pulses. Here it will be shown that (i) the written excited region can be moved by controlling pulses with frequency $\omega_{2}=\omega_{12}$; (ii) the written region can be extracted from the medium by applying a sequence of such pulses; and finally, (iii) the scaling laws for the excited regions can be extended if the writing and controlling pulses of both sequences meet the scaling conditions introduced in Sec. III. Moreover, we generalize the scaling conditions to the case of a medium prepared initially in different states, with scaled spatial distributions of the populations and coherences, as it will be further explained below.

Propagation of controlling $\omega_{2}$ pulses outside the region excited by writing pulses is weakly perturbed because of the small population of level $|2\rangle$ and because of the weak interaction of $\omega_{2}$ pulses with the transition $|1\rangle \rightarrow|3\rangle\left(\omega_{2}\right.$ pulses are far from resonance with this transition). In the excited region, however, these pulses induce transfer of the population from the excited state $|2\rangle$ to the ground state $|3\rangle$ with simultaneous emission of a pulse $\omega_{1}$. This process results in removing (cleaning) the excited region (or its main part). Now, the generated $\omega_{1}$ pulse will propagate (in some cases together with part of the reading $\omega_{2}$ pulse) and will undergo frequency-down conversion in the same manner as it was done previously by writing with the $\left(\omega_{1}, \omega_{2}\right)$ pulse. Therefore, this $\omega_{1}$ pulse will write the excited region at a new location, in such a way that the excited region can be moved either in the forward direction (by injection of $\omega_{2}$ pulses in the same direction as the initially writing pulses), or in the backward direction (by injection of $\omega_{2}$ pulses in the direction that is opposite to the propagation direction of the initially writing pulses). Such movement of the excited region in the forward direction is shown in Fig. 6(a) for a sequence of pulses with $\tau=40$ fs and in Fig. 6(b) for another sequence of pulses with $\tau=80 \mathrm{fs}$. In both sequences the area of the respective pulses is the same (see figure caption). In these figures the numbers near the curves indicate the position of the excited region induced after the propagation of the corre-

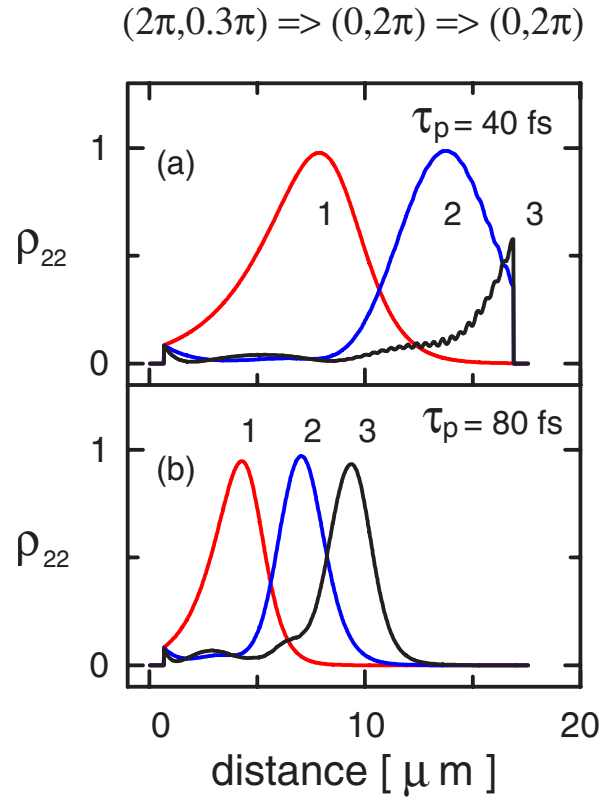

FIG. 6. (Color online) Scaling laws and possibilities of manipulation of the excited region location by pulse sequences with temporal duration of $\tau_{p}=40 \mathrm{fs}$ (a) and $\tau_{p}=80 \mathrm{fs}$ (b). Numbers indicate the position of the excited region after the propagation of the corresponding number of pulses through the medium. The pulses are as follows: the first pulse is $(2 \pi, 0.3 \pi)$, the second pulse is $(0,2 \pi)$, and the third pulse is $(0,2 \pi)$. The time delay between the subsequent pulses in the sequence is $8 \tau_{p}=320 \mathrm{fs}$ (a) and $640 \mathrm{fs}$ (b).

sponding number of pulses in the sequence. We found that the spatial width of the moved excited region is only slightly modified.

It is possible to extract the information from a written region by an appropriate sequence of pulses, as depicted in Fig. 6(a) for the particular case of pulses with a time duration such as $\tau_{p}=40 \mathrm{fs}$. In such case the last $\omega_{2}$ pulse induces transfer of population from level $|2\rangle$ into the ground state $|3\rangle$ with simultaneous appearance of a $\omega_{1}$ pulse, but the last one does not have enough time to write the excited region at a new location, and therefore this pulse leaves the medium. Hence, a spectral component at frequency $\omega_{1}$ will appear in the output spectra of the emission after the injection of the last $\omega_{2}$ pulse in the sequence. We have found that the same writing-reading procedure can be performed with pulses of different pulse area. Therefore, it has been shown that the location of the excited regions in a $\Lambda$-type quantum system can efficiently be driven by short pulses of SIT-type whose frequency is near the resonance frequency $\omega_{12}$ of the transition $|1\rangle \rightarrow|2\rangle$ [see Fig. 1(a)].

Figure 6 also demonstrates the scaling properties in the manipulation of the excited region by pulse sequences. The first pulses in each sequence write the excited regions inside the medium. From Fig. 6, it is clear that the regions excited by the first sequence of pulses [Fig. 6(a), $\tau_{p}=40 \mathrm{fs}$ ] scale with a factor 2 with respect to the regions excited by the second sequence of pulses [Fig. 6(b), $\tau_{p}=80 \mathrm{fs}$ ]. We also note that the second pulse in each sequence, and the consecutive ones, will enter a medium which is prepared with initial conditions of populations and coherences that are also scaled 
with respect to each other in their distribution in space. From Fig. 6, it follows that after the movement of the excited region, the final distributions of the populations in the two media indeed meet scaling properties [see curves 2 in Figs. 6(a) and 6(b)], which is in accordance with the scaling laws (1) and (2) under the following conditions: (i) initially, the media are prepared in states with scaled spatial distributions; (ii) the scaling factor of the distributions in the media is defined by the ratio of the temporal durations of the pulses, (iii) the area of the pulses in the two cases are equal. We found also that the spatial width of the excited region is only slightly modified during the movement, but in the same manner in each medium. The reported scaling behavior is possible because each corresponding pulse in the first and in the second sequence, as well as the considered media after interaction with each pulse, meet the scaling conditions. Therefore, we have shown that the scaling behavior is valid not only in writing the excited region by a single pulse, but also during the subsequent driving of the excited region by the pulse sequences. In our numerical calculations we also find that the validity of the scaling laws for pulse sequences has the same restrictions as those for single pulses, i.e., deviations from the scaling laws become significant at small value of the area of the pulses in the pulse sequences, namely, when it becomes on the order of $\pi$ or smaller. Hence, by means of numerical simulations we have generalized the scaling conditions and the scaling laws that were obtained for single pulse excitation and for an homogeneous space distribution of atoms (molecules) between different states to the case of excitation with sequences of pulses in media with inhomogeneous initial spatial distributions.

\section{SUMMARY AND CONCLUSIONS}

In this paper we have investigated the possibilities of coherent control in one spatial dimension of the population transfer from the ground $|3\rangle$ to the lower excited state $|2\rangle$, and the excitation of the coherence between these two states, in a three-level $\Lambda$-type system. The system is prepared initially in the ground state and is excited by one- and two-color ultrashort SIT pulses or by pulse sequences. Such pulses, whose central frequency is near resonance with the transition from the ground state [transition $|3\rangle \rightarrow|1\rangle$ in Fig. 1(a)], induce a spatial region in which population is transferred from the ground state $|3\rangle$ to the lower excited state $|2\rangle$ in a short spatial domain, which is surrounded in both sides by regions with excited coherence between the two lower states.

The excited region can be characterized by (i) location, (ii) spatial width, and (iii) maximum population transferred to the lower excited level. We have investigated the effects of different parameters of the pulses and of the medium on such excited regions. We have shown that the area of a pulse $\omega_{2}$, whose frequency is near resonance with the transition $|1\rangle$ $\rightarrow|2\rangle$, can effectively control the position of the excited region, leaving the other characteristics of the region unaffected. It has been found that the maximum value of the population of level $|2\rangle$ can be effectively controlled by the detuning of the pulse frequencies from the one-photon resonances. It has been also shown that both position and width of the excited region (with the maximum value of the population of level $|2\rangle$ being unaffected) can be controlled by varying the temporal duration of the pulses. In particular, the excited region width can be made smaller than the light wavelength. The results reported in this paper might open new possibilities for ultrafast writing, storage, reading and processing of information by optical methods on the femtosecond time scale and in nanometer spatial regions. The spatial-dependent excitation that we report may be feasible in solid-state or semiconductor materials, and also in liquids or in relatively dense gases.

The possibility of manipulating the region with spatialdependent excitations of population and coherence has been investigated under the condition that the pulse duration is less than the characteristic relaxation times of the system. We have shown that the position of the excited region can be modified by additional pulses after it is written in the medium. Indeed, it has been shown that the written region can be moved, with keeping the width of the region almost unaffected, by injection of pulses whose frequency is at resonance with the transition $|1\rangle \rightarrow|2\rangle$. Moreover, an information written in the excited region can be extracted from the medium by applying a sequence of such pulses. Finally, on the basis of our numerical simulations, we have formulated scaling laws and conditions under which different pulses with scaled amplitudes and time durations, but with the same pulse areas, will induce scaled excitation domains in the medium. It has been also demonstrated that the scaling properties can be extended to the case where the initial space distributions of the atoms (molecules) among different states meet the scaling conditions, and the excitation is provided with sequences of pulses whose durations meet the same scaling conditions.

The theoretical results obtained in this paper on coherent control of regions with medium excitation of population and coherences by SIT pulses, and the effects of the different parameters characterizing the pulses and the medium, may be used in applications of optical information processing $[20,21]$. The obtained scaling laws could be considered as a helpful tool in providing the spatial and time scale limits in some development of optical computing devices and their scaling properties.

\section{ACKNOWLEDGMENTS}

Support from the CRED-program of the Generalitat de Catalunya, from the Programa Ramón y Cajal of the Spanish Ministry of Science and Technology, and from Contract Nos. FIS2005-07931-C03-03 and FIS2005-01497 is acknowledged.

\section{APPENDIX}

Propagation of electromagnetic linearly polarized plane waves in one spatial dimension (1D), for definiteness along the $x$ axis, can be described by the Maxwell equations in a Cartesian basis as follows:

$$
\frac{\partial}{\partial t}\left(\begin{array}{l}
B_{y} \\
D_{z}
\end{array}\right)=\frac{\partial}{\partial x}\left(\begin{array}{l}
E_{z} \\
H_{y}
\end{array}\right),
$$

where $E_{z}\left(D_{z}\right)$ is the electric field (displacement), and $H_{y}\left(B_{y}\right)$ is the magnetic field (induction) vector component; $\partial / \partial t$ and $\partial / \partial x$ are the time and spatial derivatives. 
In the electric dipole approximation, the evolution of a three-level quantum system (atoms or molecules) of $\Lambda$-type [see the inset in Fig. 1(a)] driven by the propagated electromagnetic field can be described by the density matrix equations,

$$
\begin{gathered}
\partial_{t} \sigma_{11}=-\gamma_{1} \sigma_{11}-2 \Omega_{E} \operatorname{Im} \sigma_{13}-2 \Omega_{E} \beta \operatorname{Im} \sigma_{12}, \\
\partial_{t} \sigma_{22}=-\gamma_{2} \sigma_{22}+\gamma_{12} \sigma_{11}+2 \Omega_{E} \beta \operatorname{Im} \sigma_{12}, \\
\partial_{t} \sigma_{33}=+\gamma_{13} \sigma_{11}+\gamma_{23} \sigma_{22}+2 \Omega_{E} \operatorname{Im} \sigma_{13}, \\
\partial_{t} \sigma_{12}=-\left(\Gamma_{12}+i \omega_{12}\right) \sigma_{12}+i \Omega_{E}\left[\beta\left(\sigma_{11}-\sigma_{22}\right)-\sigma_{23}^{*}\right], \\
\partial_{t} \sigma_{13}=-\left(\Gamma_{13}+i \omega_{13}\right) \sigma_{13}+i \Omega_{E}\left[\left(\sigma_{11}-\sigma_{33}\right)-\beta \sigma_{23}\right], \\
\partial_{t} \sigma_{23}=-\left(\Gamma_{23}+i \omega_{23}\right) \sigma_{23}+i \Omega_{E}\left(\sigma_{12}^{*}-\beta \sigma_{13}\right),
\end{gathered}
$$

where $\sigma_{11}, \sigma_{22}$, and $\sigma_{33}$ represent the populations of levels 1 , 2 , and 3, respectively [see the inset in Fig. 1(a) and normalization below]. $\gamma_{l}=\Sigma_{m} \gamma_{l m}$, with $\gamma_{l m}$ being the decay rate of the population from the state $|l\rangle$ to the $|m\rangle$ one; $\Gamma_{l m}$ are the decay rates of the off-diagonal density matrix elements $\sigma_{l m}$ (medium polarizations); and $\omega_{l m}=\left|E_{m}-E_{l}\right| / \hbar$ are the angular frequencies of optical transitions $|l\rangle-|m\rangle$, with $E_{l, m}$ being the energies of the quantum states $|l\rangle$ and $|m\rangle$. In deriving Eqs. (A2) it was assumed that only the transitions $|1\rangle-|2\rangle$ and $|1\rangle$ -

$|3\rangle$ are allowed in the electric dipole approximation $\left(d_{23}=0\right)$.

In Eqs. (A2) the normalization of the variables is

$$
\begin{gathered}
\Omega_{\{E, D, B, H\}}=\left\{E_{z}, D_{z}, B_{y}, H_{y}\right\} d_{13} / \hbar, \\
\sigma_{l m}=\rho_{l m} N_{a} 2 d_{13}^{2} / \hbar, \\
\beta=d_{12} / d_{13},
\end{gathered}
$$

where $\rho_{l m}$ are the density matrix elements, $d_{l m}$ is the effective dipole coupling coefficient (electric dipole moment) of the optical transition $|l\rangle-|m\rangle$, and $N_{a}$ is the density of polarizable atoms. For a closed quantum system considered here the probability conservation condition $\rho_{11}+\rho_{22}+\rho_{33}=1$ applies, reducing in (A2) the number of equation for the level populations by one. When only the transitions $|1\rangle-|2\rangle$ and $|1\rangle-|3\rangle$ are allowed in the electric dipole approximation, and for nonmagnetic materials, the constitutive expressions, that complete a picture, read as follows:

$$
\begin{gathered}
\Omega_{E}=\left[\Omega_{D}+\operatorname{Re}\left(\sigma_{13}+\beta \sigma_{12}\right)\right] / \varepsilon_{0}, \\
\Omega_{B}=\Omega_{H} \mu_{0},
\end{gathered}
$$

where $\varepsilon_{0}$ and $\mu_{0}$ are the electric and magnetic constants, respectively. Equations (A1)-(A5) are investigated along this paper with a FDTD scheme that is detailed below.

Yee's discretization (staggered in space and in time) [22] for the electric and magnetic fields has been used in our modeling. On both sides the spatial grid is terminated by perfectly matched layers [23,24]. We have applied two FDTD algorithms for the solution of the density matrix and Maxwell equations, which are based on Runge-KuttaFehlberg and predictor corrector methods described in details in Refs. [14,25], respectively. Both these algorithms give the same results reported in this paper.

For definiteness, in this paper we consider propagation of single- or two-color phase-matched hyperbolic secant (HS) pulses, i.e., we will assume that the input pulse has one or two spectral components at central frequencies $\omega_{1}$ or/and $\omega_{2}$ with amplitudes $E_{\omega_{1}}$ or/and $E_{\omega_{2}}$, respectively. The wave form of such pulses at the input point $(x=0)$ can be described as follows:

$$
\begin{aligned}
E_{z}(x=0, t) & =E_{\omega_{1}}(t)+E_{\omega_{2}}(t) \\
& =E_{\omega_{1}} \frac{\cos \left[\omega_{1}\left(t-t_{0}\right)+\phi\right]}{\cosh \left[\left(t-t_{0}\right) / t_{p}\right]}+E_{\omega_{2}} \frac{\cos \left[\omega_{2}\left(t-t_{0}\right)\right]}{\cosh \left[\left(t-t_{0}\right) / t_{p}\right]} .
\end{aligned}
$$

where $\tau_{p}=2 \operatorname{arccosh}(\sqrt{2}) t_{p}$ is the full width at half-maximum (FWHM) of the pulse intensity envelope, and $t_{0}$ is the time at which the pulse envelope maximum enters the grid; $\phi$ is the initial phase difference between the $E_{\omega_{1}}$ and $E_{\omega_{2}}$ pulses. In all our simulations reported here we assumed that (1) $\phi=0 ;(2)$ the dipole moments of the transitions are equal $d_{13}=d_{12}$ $=4.2 \times 10^{-29} \mathrm{~cm}(\beta=1)$; and (3) the relaxation terms in Eqs. (A2) are disregarded, i.e., $\gamma_{l m}=\Gamma_{l m}=0$, since we consider ultrashort pulses, time durations of which are shorter than the relaxation times of the medium polarization and populations.
[1] M. Fleischhauer and A. S. Manka, Phys. Rev. A 54, 794 (1996).

[2] V. V. Kozlov and J. H. Eberly, Opt. Commun. 179, 85 (2000).

[3] S. L. McCall and E. L. Hahn, Phys. Rev. 183, 457 (1969).

[4] G. L. Lamb, Jr., Rev. Mod. Phys. 43, 99 (1971).

[5] S. E. Harris, Phys. Today 50, 36 (1997).

[6] J. P. Marangos, J. Mod. Opt. 45, 471 (1998).

[7] M. O. Scully and M. S. Zubairy, Quantum Optics (Cambridge University Press, Cambridge, 1997).

[8] M. Fleischhauer and M. D. Lukin, Phys. Rev. Lett. 84, 5094 (2000); M. D. Lukin, S. F. Yelin, and M. Fleischhauer, ibid.
84, 4232 (2000); M. Fleischhauer and M. D. Lukin, Phys. Rev. A 65, 022314 (2002).

[9] C. Liu, Z. Dutton, C. H. Behroozi, and L. V. Hau, Nature (London) 409, 490 (2001).

[10] D. F. Phillips, A. Fleischhauer, A. Mair, R. L. Walsworth, and M. D. Lukin, Phys. Rev. Lett. 86, 783 (2001).

[11] A. V. Turukhin, V. S. Sudarshanam, M. S. Shahriar, J. A. Musser, B. S. Ham, and P. R. Hemmer, Phys. Rev. Lett. 88, 023602 (2001).

[12] J. J. Longdell, E. Fraval, M. J. Sellars, and N. B. Manson, Phys. Rev. Lett. 95, 063601 (2005). 
[13] G. Vemuri, G. S. Agarwal, and K. V. Vasavada, Phys. Rev. Lett. 79, 3889 (1997).

[14] Yu. Loiko and C. Serrat, Phys. Rev. A 73, 063809 (2006).

[15] A. B. Matsko, Y. V. Rostovtsev, O. Kocharovskaya, A. S. Zibrov, and M. O. Scully, Phys. Rev. A 64, 043809 (2001).

[16] C. Mewes and M. Fleischhauer, Phys. Rev. A 66, 033820 (2002).

[17] T. N. Dey and G. S. Agarwal, Phys. Rev. A 67, 033813 (2003).

[18] M. D. Eisaman, A. André, F. Massou, M. Fleischhauer, A. S. Zibrov, and M. D. Lukin, Nature (London) 438, 837 (2005); J. J. Longdell, E. Fraval, M. J. Sellars, and N. B. Manson, Phys. Rev. Lett. 95, 063601 (2005); D. N. Matsukevich, T. Chanelière, S. D. Jenkins, S.-Y. Lan, T. A. B. Kennedy, and A.
Kuzmich, ibid. 96, 033601 (2006).

[19] S. Hughes, Phys. Rev. Lett. 81, 3363 (1998).

[20] M. D. Lukin, Rev. Mod. Phys. 75, 457 (2003).

[21] M. Fleischhauer, A. Imamoglu, and J. P. Marangos, Rev. Mod. Phys. 77, 633 (2005).

[22] K. S. Yee, IEEE Trans. Antennas Propag. 14, 302 (1966).

[23] S. D. Gedney, IEEE Trans. Antennas Propag. 44, 1630 (1996).

[24] A. Taflove and S. C. Hagness, Computational Electrodynamics: The Finite-Difference Time-Domain Method (Artech House, Boston, London, 2000).

[25] R. W. Ziolkowski, J. M. Arnold, and D. M. Gogny, Phys. Rev. A 52, 3082 (1995); G. Slavcheva, J. M. Arnold, I. Wallace, and R. W. Ziolkowski, ibid. 66, 063418 (2002). 